Pacific Journal of Mathematics

DOUBLE COMMUTANTS OF WEIGHTED SHIFTS 


\title{
DOUBLE COMMUTANTS OF WEIGHTED SHIFTS
}

\section{T. ROLF TURNER}

\begin{abstract}
Let $A$ be an operator (bounded linear transformation) on a (complex) Hilbert space. If the double commutant of $A$ is equal to the weakly closed algebra (with identity) generated by $A$ we shall say that $A$ belongs to the class $(d c)$.
\end{abstract}

By the von Neumann Double Commutant Theorem any Hermitian operator is in $(d c)$, and it is well known that any operator on a finite dimensional space is in $(d c)$. A generalization of this latter fact was proven in [10]. The object of this paper is to show that any onesided weighted shift is in $(d c)$ and that a two-sided weighted shift is in $(d c)$ if and only if it is not invertible.

In their paper "The commutants of certain Hilbert space operators", [9], Shields and Wallen show that any one-sided shift with nonzero weights generates a maximal abelian weakly closed algebra and therefore is à fortiori in $(d c)$. Related work on commutants of weighted shifts has also been done by Gellar. (See [2], [3], and [4].)

1. Definitions and notation. If $\mathfrak{S}$ is a Hilbert space we denote be $B(\mathfrak{S})$ the algebra of all operators on $\mathfrak{K}$. For $A$ belonging to $B(\mathfrak{S})$ we denote by $\mathfrak{U}_{A}$ the weakly closed algebra with identity generated by $A$, by $\mathfrak{U}_{A}^{\prime}$ the commutant of $A$, and by $\mathfrak{U}_{A}^{\prime \prime}$ the double commutant of $A$. (The reader is referred to [1, p. 1] for definitions of commutant and double commutant.) The class $(d c)$ is the class of all operators $A$ on Hilbert space such that $\mathfrak{N}_{A}=\mathfrak{U}_{A}^{\prime \prime}$.

Let $\mathfrak{S}$ be a separable Hilbert space, let $\left\{e_{n}\right\}_{n=0}^{\infty}$ be an orthonormal basis for $\mathscr{F}$, and let $\left\{\alpha_{n}\right\}_{n=1}^{\infty}$ be a bounded sequence of scalars. Define $S$ on $\mathfrak{S}$ by $S e_{n}=\alpha_{n+1} e_{n+1}$ for all $n$, extending linearly and continuously. Then $S$ is called a one-sided weighted shift on $\mathfrak{S}$.

Now let $\left\{e_{n}\right\}_{n=-\infty}^{\infty}$ be a two-side orthonormal basis for $\mathfrak{S}$ and let $\left\{\alpha_{n}\right\}_{n=-\infty}^{\infty}$ be a doubly infinite bounded sequence of scalars. Definite $S$ on $\mathfrak{S}$ by $S e_{n}=\alpha_{n+1} e_{n+1}$ for all $n$, extending linearly and continuously as before. Then $S$ is called a two-sided weighted shift on $\mathfrak{S}$.

Finally let $\mathfrak{S}_{\mathcal{C}}$ be a finite dimensional Hilbert space and let $\left\{e_{0}, \cdots, e_{k}\right\}$ be an orthonormal basis for $\mathfrak{S}$. Let $\alpha_{1} \cdots \alpha_{k}$ be scalars. Define $S$ on $\mathfrak{S}$ by $S e_{n}=\alpha_{n+1} e_{n+1}$ for $0 \leqq n<k, S e_{k}=0$, extending linearly. Then $S$ is called a finite weighted shift on $\mathfrak{S}$.

In the forthcoming we shall drop the term "weighted" and speak simply of shifts.

A backward (one-sided, two-sided, or finite) shift is the adjoint of a (one-sided, two-sided, or finite) shift respectively. Note that 
a backward finite or two-sided shift is unitarily equivalent to a (forward) finite or two sided shift respectively. The equivalence is effected simply by renumbering the basis vectors.

Notice that we do not insist that the weights of our shifts (the numbers $\alpha_{n}$ ) be nonzero.

2. Preliminaries, To facilitate our discussion of shifts we now state and prove the following lemma.

Lemma 2.1. Let $A \in B(\mathfrak{K})$ be in the class (dc). Suppose that (a) $A$ has either nontrivial kernel or co-kernel, or (b) $A$ is invertible. Let $\Re$ be a Hilbert space. Then $A \oplus 0 \in B(\mathfrak{S} \oplus \Omega)$ is in the class $(d c)$.

Proof. Case (a). It is easily seen that $A \in(d c)$ if and only if $A^{*} \in(d c)$ so we may assume without loss of generality that $A$ has nontrivial co-kernel. Let $f$ be a unit vector in co-ker $(A)$ and let $g \in \Re$. Define $X: \mathfrak{S} \rightarrow \Re$ by $X h=(h, f) \cdot g$. Then for any $h \in \mathfrak{K}, X A h=$ $(A h, f) \cdot g=0 \cdot g=0$, so $X A=0=0 X$. Now $X f=\|\mathrm{f}\|^{2} \cdot g=g$. Therefore, we conclude that $\bigcup_{X A=0 X}$ Range $X=\Omega$. This fact, together with the fact that $A \in(d c)$ is easily seen to imply that $A \oplus 0 \in(d c)$.

Case $(b)$. It is clear that $A^{-1} \in \mathfrak{X}_{A}^{\prime \prime}$. Since $A \in(d c)$ this implies that $A^{-1} \in \mathfrak{U}_{A}$, so there is a net of polynomials $\left\{p_{\alpha}\right\}$ such that $p_{\alpha}(A)$ converges to $A^{-1}$ in the weak operator topology. Therefore, $A \cdot p_{\alpha}(A)$ tends weakly to $A \cdot A^{-1}=I$. Let $q_{\alpha}(z)=z \cdot p_{\alpha}(z)$. Then $q_{\alpha}(0)=0$ so $q_{\alpha}(A \oplus 0)=q_{\alpha}(A) \oplus 0=A \cdot p_{\alpha}(A) \oplus 0$ which converges to $I \oplus 0$. We conclude that $\mathfrak{N}_{A \oplus 0}=\mathfrak{U}_{A} \oplus \mathfrak{N}_{0}$, so that $A \oplus 0 \in(d c)$.

Note that $A \in(d c)$ does not always imply that $A \oplus 0 \in(d c)$. We shall give an example to illustrate this later on.

REMARK 2.2. As previously stated we make no assumptions about the weights of our shifts being nonzero. However, any weighted shift may be expressed as a direct sum of (one-sided, two-sided, or finite) shifts having nonzero weights. Having noted this fact we proceed to treat weighted shifts by means of a case argument.

As was previously mentioned, a one-sided shift with nonzero weights is known [9, Theorem 1] to be in the class $(d c)$. A few moments reflection therefore shows that the cases we must consider are as follows:

(1) For one-sided shifts

(a) $S=\sum_{i=0}^{\infty} \oplus S_{i}$

(b) $S=\left(\sum_{i=0}^{\infty} \oplus S_{i}\right) \oplus S_{\infty}$. 
(2) For two-sided shifts

(a) $S=\left(\sum_{i=0}^{\infty} \oplus S_{i}\right) \oplus S_{\infty}$

(b) $S=\left(\sum_{i=0}^{n} \oplus S_{i}\right) \oplus S_{\infty} \oplus T$

(c) $S$ has no zero weights,

where each $S_{i}$ is a finite shift, $S_{\infty}$ is one-sided shift, and $T$ is a backward one-sided shift, all with nonzero weights.

To eliminate some redundant cases in (2) it is necessary to recall that a backward finite shift is unitarily equivalent to a (forward) finite shift.

REMARK 2.3. Application of Lemma 2.1 allows us to ignore any zero direct summands that may occur in the above.

REMARK 2.4. It is true that a two-sided shift may also assume the forms.

$$
S=\sum_{i=-\infty}^{\infty} \oplus S_{i}
$$

and

$$
S=\left(\sum_{i=0}^{\infty} \oplus S_{i}\right) \oplus T
$$

However, (i) is really the same as (1) (a) and (taking adjoints) (ii) is really the same as (2) (a).

One more preliminary observation, which we state as Lemma 2.5, is needed. Lemma 2.5 is an easy generalization of a result of Shields and Wallen [9, p. 780]. Its proof depends in turn upon an equally easy generalization of a lemma of Schur [8, Theorem V, p. 11] which Shields and Wallen employ to prove their result. (For a sketch proof of these results see also [7] Lemma 2.1, and Corollary 2.2.) We omit the proof of Lemma 2.5 here.

LEMma 2.5 Let $A \in B(\mathfrak{S})$ be a (possibly infinite) direct sum of shifts (finite, one-sided, two-sided, backward, or forward). Let $f$ be a formal power series and suppose that the matrix $f(A)$ defines an operator $D \in B(\mathfrak{E})$ (with respect to the fixed basis relative to which $A$ is realized as a direct sum of shifts). Then $D$ is in $\mathfrak{U}_{A}$.

REMARK 2.6. Because of the particularly simple form of the matrix of $A$, the matrix $f(A)$ always makes sense.

3. Main results. The way is now clear to proceed with our theorems on the double commutants of weighted shifts.

Theorem 3.1. Any one-sided shift is in the class $(d c)$.

Proof. By Remark 2.2 there are two cases to consider: 


$$
S=\sum_{i=0}^{\infty} \oplus S_{i}
$$

and

$$
S=\left(\sum_{i=0}^{n} \oplus S_{i}\right) \oplus S_{\infty}
$$

Let $S_{i}$ act on the space $\mathscr{S}_{i}$ with orthonormal basis $\left\{e_{i 0}, \cdots, e_{i n_{i}}\right\}$ and have weights $\alpha_{i 1}, \cdots, \alpha_{i n_{i}}$. Let $S_{\infty}$ act on the space $\mathfrak{F}_{\mathfrak{C}}$ with orthonormal basis $\left\{e_{i}\right\}_{i=0}^{\infty}$ and have weights $\left\{\alpha_{i}\right\}_{i=1}^{\infty}$.

Define $\pi_{i 0}=1$ and $\pi_{i j}=\prod_{k=1}^{j} \alpha_{i k}$ for $1 \leqq j \leqq n_{i}$, for $0 \leqq i<\infty$. Likewise define $\pi_{0}=1$ and $\pi_{j}=\prod_{k=1}^{j} \alpha_{k}$ for $1 \leqq j<\infty$. We now proceed to treat cases (a) and (b) separately.

Case (a). Let $D \in \mathfrak{Y}_{S}^{\prime \prime}$. It is easily seen that $D$ must have the form $D=\sum_{i=0}^{\infty} \oplus D_{i}$ where $D_{i}$ is in $\mathfrak{U}_{S_{i}}^{\prime \prime}=\mathfrak{U}_{S_{i}}$. (Since $S_{i}$ acts on a finite dimensional space it is in (dc).) Finite dimensionality also implies that $\mathfrak{U}_{S_{i}}$ is just the algebra generated by $S_{i}$, so each $D_{i}=p_{i}\left(S_{i}\right)$ where $p_{i}$ is a polynomial, in fact of degree less than or equal to $n_{i}$.

Now for any $n_{r} \geqq n_{s}$ define $E_{r s}: \mathscr{S}_{r} \rightarrow \mathscr{S}_{s}$ by

$$
E_{r s} e_{r i}= \begin{cases}\frac{\pi_{s i}}{\pi_{r i}} \cdot e_{s i} & \text { for } 0 \leqq i \leqq n_{s} \\ 0 & \text { for } n_{s}<i \leqq n_{r}\end{cases}
$$

extending linearly. A simple computation shows that $S_{s} E_{r s}=E_{r s} S_{r}$, consequently $D_{s} E_{r s}=E_{r s} D_{r}$. Now $E_{r s}$ maps $\mathscr{F}_{r}$ onto $\mathscr{S}_{s}$ so for every $h$ in $\mathfrak{S}_{r}$ there is a $g$ such that $h=E_{r s} g$. Therefore, $D_{s} h=D_{s} E_{r s} g=$ $E_{r s} D_{r} g=E_{r s} p_{r}\left(S_{r}\right) g=p_{r}\left(S_{s}\right) E_{r s} g=p_{r}\left(S_{s}\right) h$. We conclude that $p_{s}\left(S_{s}\right)=$ $D_{s}=p_{r}\left(S_{s}\right)$ for $n_{r} \geqq n_{s}$.

If the sequence $\left\{n_{i}\right\}_{i=0}^{\infty}$ is bounded we may choose $n_{r}=\sup _{i} n_{i}$. Then for every $i, D_{i}=p_{i}\left(S_{i}\right)=p_{r}\left(S_{i}\right)$ so that $D=p_{r}(S) \in \mathfrak{A}_{s}$.

If the sequence $\left\{n_{i}\right\}_{i=0}^{\infty}$ is unbounded we define a formal power series

$$
f(z)=\sum_{k=0}^{\infty} \gamma_{k} z^{k} \text { where } \gamma_{k}
$$

is the $k$ th coefficient of any $p_{i}$ with $n_{i} \geqq k$. Since $n_{r} \geqq n_{s}$ implies $p_{r}\left(S_{s}\right)=p_{s}\left(S_{s}\right)$ we see that the $k$ th coefficients of $p_{r}$ and $p_{s}$ agree whenever $n_{r}$ and $n_{s}$ are both greater than or equal to $k$, so the numbers $\gamma_{k}$ are well-defined.

Now consider $f(S)$, the formal power series in $S$. For each $i, S_{i}$ is nilpotent of order $n_{i}+1$ and the coefficients of $f$ of index less than or equal to $n_{i}$ agree with those of $p_{i}$. Thus $f\left(S_{i}\right)=p_{i}\left(S_{i}\right)=D_{i}$. Therefore, the matrix $f(S)$ defines the operator $D$, so by Lemma 2.5 $D$ is in $\mathfrak{U}_{S}$. 
Case (b). If $D$ is in $\mathfrak{U}_{S}^{\prime \prime}$ then $D=\sum_{i=0}^{\infty} \oplus D_{i} \oplus D_{\infty}$ where each $D_{i}$ is in $\mathfrak{A}_{S_{i}}^{\prime \prime}, i=0, \cdots, N, \infty$. Each $D_{i}, 0 \leqq i \leqq N$, is a polynomial $p_{i}$ in $S_{i}$ and $D_{\infty}$ is a formal power series $g$ in $S_{\infty}$. (See [6, p. 780].) Define $E_{r}: \mathfrak{K} \rightarrow \mathfrak{S}_{r}$ by

$$
E_{r} e_{i}= \begin{cases}\frac{\pi_{r i}}{\pi_{i}} \cdot e_{r i} & \text { for } \quad 0 \leqq i \leqq n_{r} \\ 0 & \text { for } \quad i>n_{r}\end{cases}
$$

extending linearly. By arguments similar to those used for case (a) we can show that the coefficients of the polynomials $p_{i}$ agree (as far as they go) with those of $g$, whence $D=g(S)$ and so is in $\mathfrak{U}_{S}$.

We now turn to the discussion of two-sided shifts. These operators need not always be in the class $(d c)$. The familiar bilateral shift (all weights equal to 1) provides a counter-example. If we denote this shift by $S$, then $S^{*}=S^{-1}$ is in $\mathfrak{U}_{S}^{\prime \prime}$ but not in $\mathfrak{A}_{S}$.

As it turns out, this example gives the clue to the general situation. If a two-sided shift $S$ is invertible then $S^{-1} \in \mathfrak{P}_{S}^{\prime \prime}$ but $S^{-1} \notin \mathfrak{A}_{S}$. If $S$ is not invertible then $\mathfrak{U}_{S}=\mathfrak{U}_{S}^{\prime \prime}$.

Proposition 3.2. If $S$ is a two-sided shift with at least one zero weight then $S \in(d c)$.

Proof. By Remark 2.2 we have two cases to consider:

(a) $S=\left(\sum_{i=0}^{\infty} \oplus S_{i}\right) \oplus S_{\infty}$. This case can be treated by exactly the same methods as were used in case (b) of Theorem 3.1.

(b) $S=\left(\sum_{i=0}^{N} \oplus S_{i}\right) \oplus S_{\infty} \oplus T$. If we can show that an operator in the double commutant of $S_{\infty} \oplus T$ is a formal power series in $S_{\infty} \oplus T$, then Lemma 2.5 and the same arguments as were used in Theorem 3.1, part (b), will show that $S \in(d c)$. We therefore turn our attention to operators of the form $R \oplus T$ where $R$ is a forward and $T$ a backward one-sided shift, both with nonzero weights.

Let $R$ act on $\mathfrak{S}$ with orthonormal basis $\left\{e_{i}\right\}_{i=0}^{\infty}$ and have weights $\left\{\alpha_{i}\right\}_{i=1}^{\infty}$. Let $T$ act on $\Re$ with orthonormal basis $\left\{f_{i}\right\}_{i=0}^{\infty}$ and have weights $\left\{\beta_{i}\right\}_{i=1}^{\infty}$.

$$
\begin{aligned}
& \text { Define } \pi_{0}=1, \pi_{j}=\prod_{k=1}^{j} \alpha^{k}, \text { for } 1 \leqq j<\infty . \\
& \text { Define } \kappa_{0}=1, \kappa_{j}=\prod_{k=1}^{j} \beta_{k}, \text { for } 1 \leqq j<\infty .
\end{aligned}
$$

Let $D$ be in the double commutant of $R \oplus T$. Then $D=E \oplus F$ with $E$ in $\mathfrak{U}_{R}^{\prime \prime}=\mathfrak{A}_{R}$ and $F$ is $\mathfrak{U}_{T}^{\prime \prime}=\mathfrak{A}_{T}$. By [6, p. 780] $E$ is a formal power series $f(R)$ in $R$. Likewise $F^{*}$ is a formal power series in $T^{*}$ 
so $F$ is a formal power series $g(T)$ in $T$. If we can show that the coefficients of $g$ agree with those of $f$ then we will know that $D$ is a formal power series $f(R \oplus T)$.

To demonstrate this agreement of coefficients define operators $G_{r}: \mathfrak{S} \rightarrow \Re$ by means of the matrix $\left[\gamma_{i j}\right]$ where

$$
\gamma_{i j}=\left\{\begin{array}{cll}
\frac{1}{\kappa_{i} \pi_{j}} & \text { for } & i+j \leqq r \\
0 & \text { for } & i+j>r
\end{array}\right.
$$

At most finitely many of the numbers $\gamma_{i j}$ are nonzero so this matrix defines an operator. It is easily seen that $T G_{r}=G_{r} R$ and that $G_{r}$ maps the span of $\left\{e_{0}, \cdots, e_{r}\right\}$ onto the span of $\left\{f_{0}, \cdots, f_{r}\right\}$.

Now write $E=f(R)=\sum_{i=0}^{\infty} \mu_{i} R^{i}$. (The series converges on finite linear combinations of basis vectors.) Likewise write $F=g(T)=$ $\sum_{i=0}^{\infty} \nu_{i} T^{i}$. Then, for any $r \geqq 0$,

$F f_{r}=F G_{r} h$ for some $h$ in the span of $\left\{e_{0}, \cdots, e_{r}\right\}$

$$
\begin{aligned}
& =G_{r} E h \\
& =G_{r} f(R) h \\
& =\sum_{i=0}^{\infty} \mu_{i} G_{r} R^{i} h \\
& =\sum_{i=0}^{\infty} \mu_{i} T^{i} G_{r} h \\
& =\sum_{i=0}^{\infty} \mu_{i} T^{i} f_{r} \\
& =\sum_{i=0}^{r} \mu_{i} \frac{\kappa_{r}}{\kappa_{i}} f_{r-i} .
\end{aligned}
$$

On the other hand $F f_{r}=g(T) f_{r}$

$$
\begin{aligned}
& =\sum_{i=0}^{\infty} \nu_{i} T^{i} f_{r} \\
& =\sum_{i=0}^{r} \nu_{i} \cdot \frac{\kappa_{r}}{\kappa_{i}} f_{r-i} .
\end{aligned}
$$

Comparing coefficients we see that $\nu_{r}=\mu_{r}$ for all $r$.

Proposition 3.3. Let $S$ be a two-sided shift with nonzero weights $\left\{\alpha_{i}\right\}_{i=-\infty}^{\infty}$. Suppose that $\inf _{i}\left|\alpha_{i}\right|=0$. Then $S \in(d c)$; in fact $S$ generates a maximal abelian weakly closed algebra.

Proof. Let $C \in \mathfrak{A}_{S}^{\prime}$ with matrix $\left[\gamma_{i j}\right]$. Computing $S C e_{j}=C S e_{j}$ for arbitrary $j$ and comparing coefficients we obtain the following characterization for the numbers $\gamma_{i j}$ : 


$$
\gamma_{i j}=\left\{\begin{array}{l}
\frac{\alpha_{j+1} \times \cdots \times \alpha_{0}}{\alpha_{i+1} \times \cdots \times \alpha_{i-j}} \gamma_{i-j 0} \text { for } j \leqq 0 \\
\frac{\alpha_{i-j+1} \times \cdots \times \alpha_{i}}{\alpha_{1} \times \cdots \times \alpha_{j}} \gamma_{i-j 0} \text { for } j>0 .
\end{array}\right.
$$

An easy but tedious case argument shows that this is equivalent to

$$
\gamma_{i j}= \begin{cases}\frac{\alpha_{j+1} \times \cdots \times \alpha_{i}}{\alpha_{1} \times \cdots \times \alpha_{i-j}} \gamma_{i-j 0} & \text { for } i \geqq j \\ \frac{\alpha_{i-j+1} \times \cdots \times \alpha_{0}}{\alpha_{i+1} \times \cdots \times \alpha_{j}} \gamma_{i-j 0} & \text { for } i<j .\end{cases}
$$

Now fix a positive integer $k$ and consider the $k$ th super-diagonal $i=j-k$. Along this super-diagonal

$$
\gamma_{i j}=\frac{\alpha_{0} \times \cdots \times \alpha_{-k+1}}{\alpha_{j-k+1} \times \cdots \times \alpha_{j}} \gamma_{-k 0} .
$$

But

$$
\inf _{j}\left|\alpha_{j-k+1} \times \cdots \times \alpha_{j}\right| \leqq \inf _{j}\|S\|^{k-1}\left|\alpha_{j}\right|=\|S\|^{k-1} \inf _{j}\left|\alpha_{j}\right|=0 .
$$

Therefore the numbers $\gamma_{i j}$ are unbounded along this super-diagonal unless $\gamma_{-k 0}=0$. Since $C$ is an operator we must indeed have $\gamma_{-k 0}=0$, whence the whole kth super-diagonal is zero.

This holds for $k=1,2,3, \cdots$. We conclude that $C$ is the formal power series in $S, \sum_{i=0}^{\infty} \mu_{i} S^{i}$ where $\mu_{0}=\gamma_{00}$, and $\mu_{i}=\gamma_{i 0} /\left(\alpha_{1} \times \cdots \times \alpha_{i}\right)$ for $i>0$. By Lemma 2.5, $C \in \mathfrak{A}_{S}$.

THEOREM 3.4. A two-sided shift is in (dc) if and only if it is not invertible.

Proof. If $S$ is an invertible two-sided shift with weight sequence $\left\{\alpha_{i}\right\}_{i=-\infty}^{\infty}$, then $S^{-1}$ is the backward shift with weight sequence $\left\{1 / \alpha_{i}\right\}_{i=-\infty}^{\infty}$. Since $S^{-1}$ is a backward shift, its matrix is strictly upper triangular and hence it cannot be in $\mathfrak{U}_{S}$. However, $S^{-1}$ is clearly in $\mathfrak{U}_{S}^{\prime \prime}$.

On the other hand, if $S$ is not invertible the infimum of the moduli of its weights is zero. By Propositions 3.2 and $3.3 S \in(d c)$.

4. Concluding remarks. We may use the above theorem to construct counter-examples to some fairly reasonable-sounding conjectures about the class $(d c)$.

First of all we show that the direct sum of operators in $(d c)$ need not be in $(d c)$ : Let $S$ be a two-sided shift with nonzero weights, the infimum of the moduli of which is zero. Since $S$ has zero kernel and dense range, $S$ and the zero operator cannot be intertwined. Thus the commutant of $S \oplus 0$ is $\mathfrak{N}_{S}^{\prime} \oplus \mathfrak{R}_{0}^{\prime}$, so the double commutant is 
$\mathfrak{A}_{S}^{\prime \prime} \oplus \mathfrak{X}_{0}^{\prime \prime}=\mathfrak{A}_{S} \oplus \mathfrak{A}_{0}$. Consequently $I \oplus 0$ is in the double commutant of $S \oplus 0$, but it is clearly not in $\mathfrak{A}_{s \oplus 0}$, so $S \oplus 0 \notin(d c)$. However, 0 clearly is in $(d c)$ and $S \in(d c)$ by Theorem 3.4. (This furnishes the example promised after Lemma 2.1.)

Secondly we show that $(d c)$ is not preserved under norm limits. The operator $S$ in the previous example may be taken to be compact. (Let the weights of $S$ tend to 0 in both directions.) Then $S \oplus 0$ is compact also, so $S \oplus 0$ is the limit in norm of finite rank operators. Any finite rank operator belongs to $(d c)$, but $S \oplus 0$ does not.

To see that finite rank operators are all in $(d c)$, let $F$ be finite rank and write $F$ as $E \oplus 0$ where $E$ acts on a finite dimensional space. The operator $E \in(d c)$ and is either invertible or has nonzero kernel. By Lemma 2.1 $F \in(d c)$.

\section{REFERENCES}

1. J. Dixmier, Les Algebres d'Opérateurs dans l'Espace Hilbertien, Gauthier-Villars, Paris, (1969).

2. R. Gellar, Shift Operators in Banach Space, Dissertation, Columbia University, 1968. 3. — Operators commuting with a weighted shift, Proc. Amer. Math. Soc., 23 (1969), 538-545.

4. - Two sublattices of weighted shift invariant subspaces, to appear.

5. P. R. Halmos, A Hilbert Space Problem Book, Van Nostrand, Princeton, (1967).

6. N. Jacobson, Lectures in Abstract Algebra, Volume II, Van Nostrand, Princeton (1953).

7. A. L. Lambert, The algebra generated by an invertibly weighted shift (to appear).

8. I. Schur, Bemerkungen zur Theorie der beschraenkten Bilinearformen mit unendlich vielen Veraenderlichen, J Reine Angew. Math., 140 (1911), 1-28.

9. A. L. Shields and L. J. Wallen, The commutants of certain Hilbert space operators, Indiana Univ. Math. J., 20 (1971), 777-788.

10. T. R. Turner, Double commutants of algebraic operators, to appear, Proc. Amer. Math. Soc.

Received December 1, 1971 and in revised form February 11, 1972. This research constitutes part of the author's doctoral dissertation, written at the University of Michigan under the direction of Carl M. Pearcy.

University of Alberta, Edmonton, Alberta, Canada 


\section{PACIFIC JOURNAL OF MATHEMATICS}

EDITORS

\author{
H. SAMELSON \\ Stanford University \\ Stanford, California 94305 \\ C. R. HOBBY \\ University of Washington \\ Seattle, Washington 98105
}

\author{
J. DUGUNDJI \\ Department of Mathematics \\ University of Southern California \\ Los Angeles, California 90007 \\ RICHARD ARENS \\ University of California \\ Los Angeles, California 90024
}

\section{ASSOCIATE EDITORS}

E. F. BECKENBACH

B. H. NeUManN

F. WOLF

K. YosHIDA

\section{SUPPORTING INSTITUTIONS}

UNIVERSITY OF BRITISH COLUMBIA

CALIFORNIA INSTITUTE OF TECHNOLOGY

UNIVERSITY OF CALIFORNIA

MONTANA STATE UNIVERSITY

UNIVERSITY OF NEVADA

NEW MEXICO STATE UNIVERSITY

OREGON STATE UNIVERSITY

UNIVERSITY OF OREGON

OSAKA UNIVERSITY
UNIVERSITY OF SOUTHERN CALIFORNIA

STANFORD UNIVERSITY

UNIVERSITY OF TOKYO

UNIVERSITY OF UTAH

WASHINGTON STATE UNIVERSITY UNIVERSITY OF WASHINGTON

AMERICAN MATHEMATICAL SOCIETY NAVAL WEAPONS CENTER 


\section{Pacific Journal of Mathematics}

\section{Vol. 45, No. $1 \quad$ September, 1973}

William George Bade, Complementation problems for the Baire classes .......... 1

Ian Douglas Brown, Representation of finitely generated nilpotent groups ........ 13

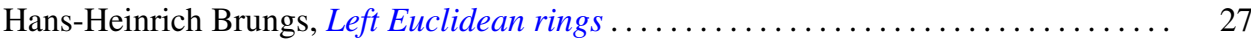

Victor P. Camillo and John Cozzens, A theorem on Noetherian hereditary rings ..... 35

James Cecil Cantrell, Codimension one embeddings of manifolds with locally flat

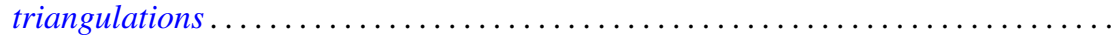

L. Carlitz, Enumeration of up-down permutations by number of rises . . . . . . . . . .

Thomas Ashland Chapman, Surgery and handle straightening in Hilbert cube

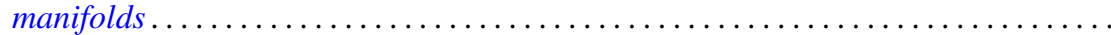

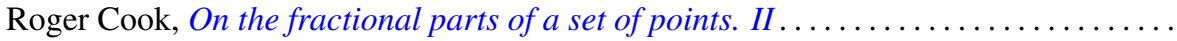

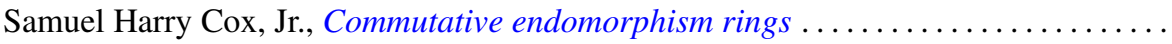

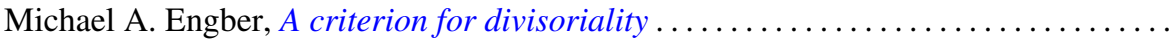

Carl Clifton Faith, When are proper cyclics injective . . . . . . . . . . . . . . 97

David Finkel, Local control and factorization of the focal subgroup . . . . . . . . . 113

Theodore William Gamelin and John Brady Garnett, Bounded approximation by

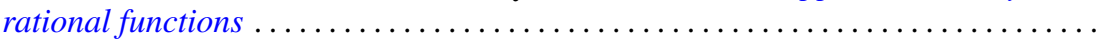

Kazimierz Goebel, On the minimal displacement of points under Lipschitzian

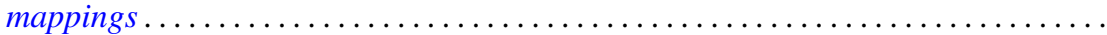

Frederick Paul Greenleaf and Martin Allen Moskowitz, Cyclic vectors for representations associated with positive definite measures: nonseparable

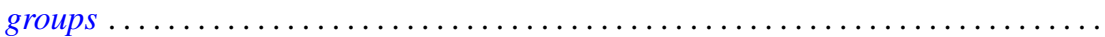

Thomas Guy Hallam and Nelson Onuchic, Asymptotic relations between perturbed linear systems of ordinary differential equations .

David Kent Harrison and Hoyt D. Warner, Infinite primes of fields and completions. .

James Michael Hornell, Divisorial complete intersections . ......

Jan W. Jaworowski, Equivariant extensions of maps ..............

John Jobe, Dendrites, dimension, and the inverse arc function .. .

Gerald William Johnson and David Lee Skoug, Feynman integrals of non-factorable

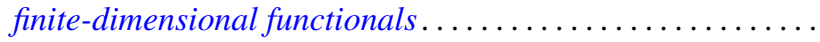

Dong S. Kim, A boundary for the algebras of bounded holomorphic functions ...... 269

Abel Klein, Renormalized products of the generalized free field and its derivatives ... 275

Joseph Michael Lambert, Simultaneous approximation and interpolation in $L_{1}$ and

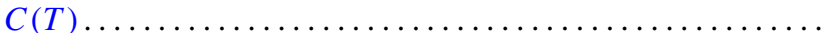

Kelly Denis McKennon, Multipliers of type $(p, p)$ and multipliers of the group

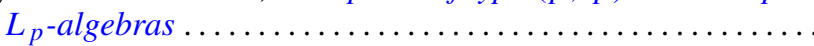

William Charles Nemitz and Thomas Paul Whaley, Varieties of implicative

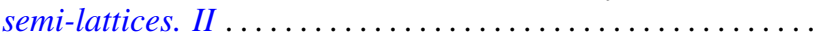

Donald Steven Passman, Some isolated subsets of infinite solvable

Norma Mary Piacun and Li Pi Su, Wallman compactifications on E-completely

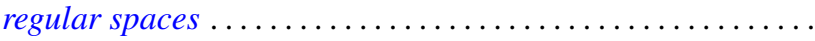

Jack Ray Porter and Charles I. Votaw, $S(\alpha)$ spaces and regular Hausdorff extensions....

Gary Sampson, Two-sided $L_{p}$ estimates of convolution transforms .

Ralph Edwin Showalter, Equations with operators forming a rig
Raymond Earl Smithson, Fixed points in partially ordered sets .

Victor Snaith and John James Ucci, Three remarks on symmetric products and

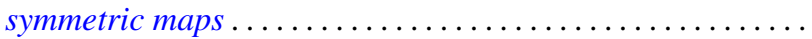

